\title{
Espacialização temporal dos focos de queimadas e de poluentes atmosféricos (Co, Ch4, No2, N2o) em Alagoas
}

\author{
Temporal spacialization of biomass burning and atmospheric \\ pollutants $(\mathrm{CO}, \mathrm{CH} 4, \mathrm{NO} 2, \mathrm{~N} 2 \mathrm{O})$ in Alagoas
}

\author{
Ronabson Cardoso Fernandes ${ }^{1}$, Washington Luiz Félix Correia Filho ${ }^{2}$ \\ ${ }^{1}$ Universidade Federal de Alagoas - UFAL, Instituto de Ciências Atmosférica - ICATCidade Universitária - Maceió - AL, Brasil \\ ${ }^{2}$ Universidade Federal do Rio Grande do Norte- UFRN, Programa de Pós-Graduação em Ciências Climáticas - Lagoa Nova, \\ Natal - RN, Brasil
}

\begin{abstract}
Resumo
A queimada é um processo de queima da biomassa, levando a produção de água, poluentes atmosféricos e de gases de efeito estufa. Com isso, localizar e espacializar esses focos é de extrema importância para o seu entendimento e suas consequências no meio ambiente e na saúde humana. Para isso, necessitou a utilização de satélites NOAA 12, 14, 15, 16, 17 e 18, GOES 08 e 12, AQUA-01, TERRA-01 e MMODIS-01, disponibilizados pelo CPTEC-INPE, durante os anos de 2004 a 2009. Paralelamente, realizaram-se estimativas de emissão anual de $\mathrm{CO}, \mathrm{NO}_{2}, \mathrm{CH}_{4}$ e $\mathrm{N}_{2} \mathrm{O}$, a partir da produção anual da cana-de-açúcar. Para a elaboração dos mapas, foi empregado o software SURFER 8.0. Utilizaram-se o Mapa de Ponto para distribuição dos focos pontuais, e o Mapa de Contorno para espacialização dos pontos interpolados, pelo método geoestatístico Krigging. Os resultados obtidos mostraram que as maiores incidências de focos de queimadas ocorreram na região canavieira, entre o litoral e o agreste alagoano, de setembro a fevereiro, período da colheita da cana-de-açúcar. Em relação à emissão de poluentes atmosféricos, o município de Coruripe e São Miguel dos Campos foram os maiores emissores de poluentes $\left(\mathrm{CO}, \mathrm{NO}_{2}\right)$ e de gases de efeito estufa em Alagoas $\left(\mathrm{CH}_{4}, \mathrm{~N}_{2} \mathrm{O}\right)$. Portanto, nas regiões produtoras de cana-de-açúcar, têm-se as maiores incidências de focos de queimadas e, consequentemente, maiores emissões de poluentes atmosféricos e de gases de efeito estufa.
\end{abstract}

Palavras-chave: cana-de-açúcar, satélites, gases de efeito estufa.

\begin{abstract}
Burning is a process of burning biomass, leading to production of water, air pollutants and greenhouse gases. So, locating and spatializing these outbreaks is extremely important for their understanding and their consequences on the environment and human health. For this, it was necessary the use of NOAA 12, 14, 15, 16, 17 and 18, GOES 8 and 12, AQUA-01, TERRA-01 and MMODIS-01 satellites, provided by CPTEC-INPE, between 2004 and 2009. In parallel, annual emissions of CO, NO2, CH4 and N2O were carried out from annual production of sugarcane. In relation to air pollutants emissions, São Miguel and Coruripe were the largest emitters of pollutants (CO, NO2) and greenhouse gases in Alagoas $(\mathrm{CH} 4, \mathrm{N2O})$. Therefore, in regions producing sugarcane, have been the highest incidence of fire focuses and consequently higher emissions of air pollutants and greenhouse gases.
\end{abstract}

Keywords: sugarcane, satellites, greenhouse gases. 


\section{Introdução}

Segundo Freitas et al. (2005), a queimada é um processo de queima de biomassa, produzindo principalmente água e dióxido de carbono. No entanto, devido à queima incompleta da matéria orgânica, leva a emissão de poluentes atmosféricos como o monóxido de carbono (CO), o dióxido de nitrogênio $\left(\mathrm{NO}_{2}\right)$ e partículas inaláveis (PTS), além dos gases de efeito estufa (GEEs) como o metano $\left(\mathrm{CH}_{4}\right)$, óxido nítrico $\left(\mathrm{N}_{2} \mathrm{O}\right)$ e o dióxido de carbono $\left(\mathrm{CO}_{2}\right)$ (FREITAS et al., 2005; LARA et al., 2005).

A prática das queimadas tem sido utilizada corriqueiramente no processo de limpeza de terreno para baratear a colheita na agilização da colheita da cana-de-açúcar, entre outras agropastoris, na substituição da vegetação nativa agropastoris, levando à degeneração do solo e da qualidade do ar (LARA et al., 2005; RIBEIRO, 2008; RONQUIM,2010).

Segundo o levantamento realizado por Lima et al. (1999), a cana-de-açúcar é responsável por cerca de $98 \%$ das emissões de gases provenientes da queima de resíduos agrícolas no Brasil, considerando-se que o Brasil se destaca como o maior produtor e exportador de derivados de cana-de-açúcar (RONQUIM, 2010). Essa cultura e indústria canavieiras são muito importantes para o desenvolvimento econômico de Alagoas, com participação de $45 \%$ do Produto Interno Bruto (PIB) Alagoano.

No Brasil, os focos de queimadas têm sido monitorados diariamente pelos satélites de órbita polar e geoestacionário, disponibilizados com excelência pelo site do Centro de Previsão de Tempo e Estudos Climáticos - Instituto Nacional de Pesquisas Espaciais (CPTEC - INPE), e que contribuem, assim, trazer ao conhecimento as queimadas não somente em Alagoas, como em todo Brasil.

Assim, o objetivo deste trabalho foi estudar os focos de queimadas em Alagoas, no período de 2004 a 2009, e suas emissões.

\section{Procedimentos}

\subsection{Localização}

Situado na Região Nordeste do Brasil, o estado de Alagoas possui uma área territorial de 27.993 km², correspondendo a $1,7 \%$ da área da Região Nordeste, situado entre as latitudes 350. $09^{\prime} 09^{\prime \prime} \mathrm{W}$ e $38^{\circ} 14^{\prime} 27^{\prime \prime} \mathrm{W}$, e longitudes $08^{\circ} 48^{\prime} 47^{\prime \prime} \mathrm{S}$ e $10^{\circ} 30^{\prime} 09^{\prime \prime} \mathrm{S}$. Delimita-se ao norte com Pernambuco, ao sul com Sergipe e a sudoeste com a Bahia, banhado pelo Oceano Atlântico (Figura 1).

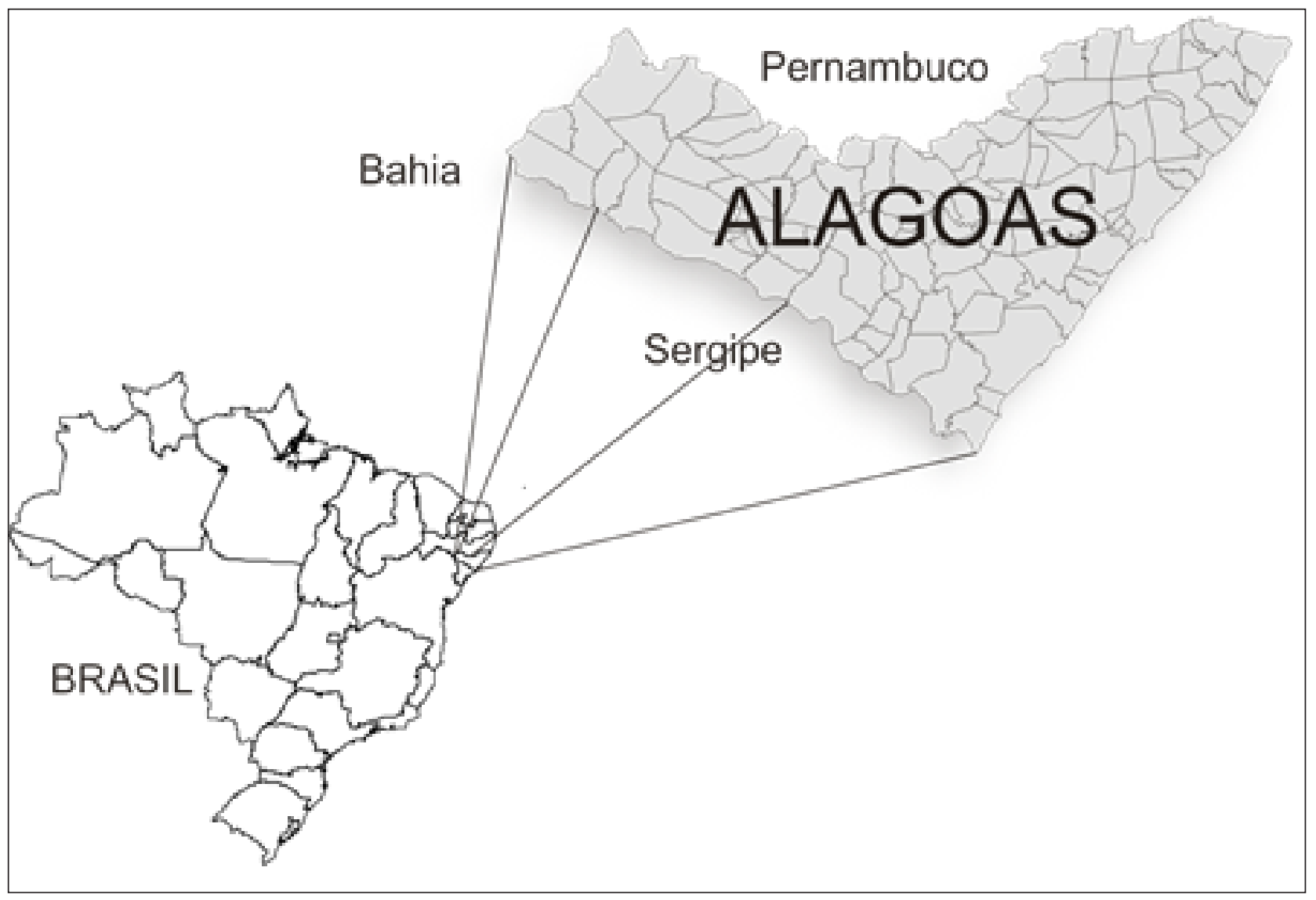

Figura 1 - Localização do Estado de Alagoas. 


\subsection{Tratamento dos dados}

Para esse estudo, foram utilizados todos os dados brutos detectados pelos satélites ambientais NOAA 12, 14, 15, 16, 17 e 18, GOES 08 e 12, AQUA01, TERRA-01 e MMODIS-01, disponibilizados pelo CPTEC-INPE através do site http://sigma.cptec.inpe.br/ queimadas/, no período de 2004 a 2009, para o Estado de Alagoas. A resolução desses satélites varia de acordo com a órbita de cada um deles. O pixel corresponde a $1 \mathrm{~km} \times 1 \mathrm{~km}\left(1 \mathrm{~km}^{2}\right)$ e, para os de órbitas polares, é de $4 \mathrm{~km} \times 4 \mathrm{~km}\left(16 \mathrm{~km}^{2}\right)$ para os geoestacionários.

Os dados obtidos foram organizados, separados e acumulados mensalmente, bimestralmente e anualmente, e, posterior a isso, foram feitas as médias e porcentagens. Para a interpolação dos dados, foram separados e acumulados todos os dados por município (coordenadas: longitude e latitude) no período em estudo.

Para a elaboração dos mapas, empregou-se o software SURFER 8.0, pelo método geoestatístico Krigging. Para a distribuição dos focos de queimadas pontuais, utilizaram-se o Mapa de Ponto (Post Map) e o Mapa de Contorno (Contour Map), para espacialização dos pontos interpolados. Este método geoestatístico Krigging leva em consideração as características espaciais de autocorrelação de variáveis regionalizadas. Isto permite que os dados obtidos por amostragem de certos pontos possam ser usados para parametrizar a estimação de pontos onde o valor da variável seja desconhecido onde o valor interpolado para qualquer nó pelo semivariograma (1), segundo Vieira (2000) e Silva et al. (2011).

$$
\gamma(h)=\frac{1}{2 N(h)} \sum_{i=1}^{N(h)}\left\{z\left(x_{i}\right)-z\left(x_{i}+h\right)\right\}^{2}
$$

$\gamma(h)$ corresponde à semivariância estimada para uma distância $h$, considerando-se $\mathrm{N}(\mathrm{h})$ os números de pares amostrais da precipitação(z) separados por uma distância $h, x_{i}$ e $x_{i}+h$ pontos dessas amostragens na distância (h) e valores de precipitação medidos nesses locais, $z\left(x_{i}\right)$ e $z\left(x_{i}+h\right)$.

Os resultados obtidos pelo variograma foram autoajustados pelo método linear (2).

$$
\gamma(h)=c_{0}+\frac{c_{1}}{a}
$$

Foi considerado o efeito pepita $\left(c_{0}\right)$, a variância estrutural $\left(c_{1}\right)$ e o alcance $(a)$.

\subsection{Emissão de gases}

Para o cálculo da emissão de gases devido à queimada, em Alagoas, utilizaram-se dados municipais de produção, área, e rendimento da cana-de-açúcar (in natura/crua) provenientes do Instituto Brasileiro de Geografia e Estatística (IBGE, 2013), no período de 2004 a 2009. Os valores da equação (3) basearam-se nos resultados obtidos por Lima et al. (2010), e conferidos posteriormente.

$$
M c=\left(P \times R \times R_{S} \times R_{e} \times R_{q}\right) \times T c
$$

$\mathrm{M}_{\mathrm{C}}=$ massa total do carbono ou nitrogênio;

$\mathrm{P}=$ produção vegetal anual, em gigagramas $\left(\mathrm{Gg}=10^{9}\right.$ gramas);

$\mathrm{R}$ = relação resíduo produção;

$\mathrm{R}_{\mathrm{S}}$ = conteúdo de matéria seca do resíduo;

$\mathrm{R}_{\mathrm{e}}=$ proporção de resíduos expostos ã queima;

$\mathrm{R}_{\mathrm{q}}=$ fração oxidada de resíduos durante a queima;

$\mathrm{T}_{\mathrm{c}}=$ conteúdo de carbono nos resíduos.

A massa total de nitrogênio $(\mathrm{MN})$ produzida durante a queimada é obtida, multiplicando-se a massa $\mathrm{MC}$ pela razão N/C. As quantidades de $\mathrm{CO}, \mathrm{CH}_{4}, \mathrm{NO}$ e $\mathrm{NO}_{2}$ foram estimadas a partir da multiplicação das taxas de emissão e fatores de conversão para cada gás, apresentados na Tabela 1, pelas massas MC ou MN, caso o gás seja composto de carbono ou nitrogênio, respectivamente.

Tabela 1- Taxas de emissão de gases liberados durante a queima de resíduos agrícolas e fatores de conversão para o cálculo das emissões, segundo IPCC.

\begin{tabular}{ccc}
\hline Gás & Taxa de emissão & Fator de conversão \\
\hline CH4 & $0,005(\mathrm{C})$ & $16 / 12$ \\
$\mathrm{CO}$ & $0,060(\mathrm{C})$ & $28 / 12$ \\
$\mathrm{~N} 2 \mathrm{O}$ & $0,007(\mathrm{~N})$ & $44 / 28$ \\
$\mathrm{NO} 2$ & $0,121(\mathrm{~N})$ & $46 / 14$ \\
\hline
\end{tabular}

Fonte: LIMA et al., (2010).

Para essas taxas de emissões, em unidades de carbono (C) ou nitrogênio (N), necessitou-se empregar fatores de conversão, considerando o peso molecular dos gases emitidos. E, por fim, a emissão desses gases foi calculada segundo a sequência abaixo:

Emissão de $\mathrm{CO}=$ carbono liberado $\mathrm{x}$ taxa de emissão x fator de conversão; 
Emissão de $\mathrm{CH}_{4}=$ carbono liberado $x$ taxa de emissão $x$ fator de conversão;

Emissão de $\mathrm{N}_{2} \mathrm{O}$ = carbono liberado $x$ razão $\mathrm{N} / \mathrm{C} x$ taxa de emissão $x$ fator de

Emissão de $\mathrm{NO}_{2}=$ (biomassa de resíduos queimada) $x$ Tc $x$ razão N/C $x$ taxa de emissão $x$ fator de conversão

Baseando-se nesses cálculos, foram elaborados mapas para estudo desses poluentes e gases de efeito estufa para Alagoas.

\section{Resultados e Discussão}

A Figura 2 mostra a distribuição de acumulados bimestrais dos focos de queimadas no Estado de Alagoas, do período de 2004 a 2009. Nota-se que os focos, em Alagoas, diminuem significativamente
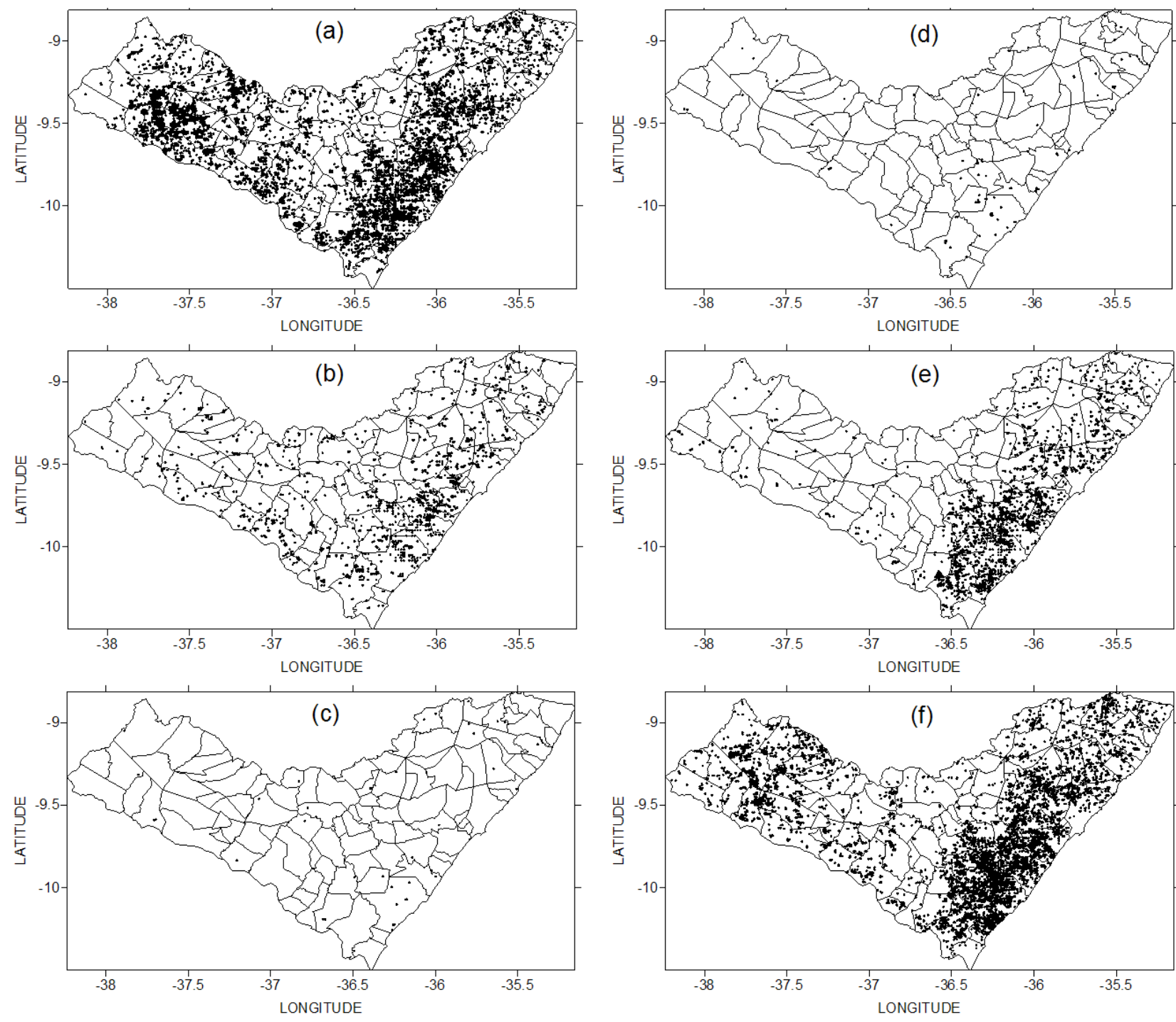

entre os meses de janeiro a junho (Fig. 2 a,b,c), pois compreende o período chuvoso de Alagoas. Segundo Cardim (2003) e Carvalho (2010), o início da estação chuvosa nessa região começa no primeiro decêndio de abril, com o término no segundo decêndio de outubro. Contudo, o período de julho a dezembro tem comportamento inverso (Fig. 2 d,e,f).

Verificou-se, também, que grande parte desses focos situa-se no litoral e outra parte na região do sertão alagoano, onde ocorre a colheita da cana-de-açúcar de setembro a fevereiro. No litoral, a prática da queima da palha da cana-de-açúcar é comum para agilizar o processo de colheita canavieira. Enquanto, na região do sertão, esta prática deve-se pela situação climatológica, com longos períodos de estiagem, baixa umidade e altas temperaturas.

A figura $3(\mathrm{a}, \mathrm{b}, \mathrm{c}, \mathrm{d}, \mathrm{e}, \mathrm{f})$ representa a produção de cana-de-açúcar no período de 2004 a 2009 em tone-

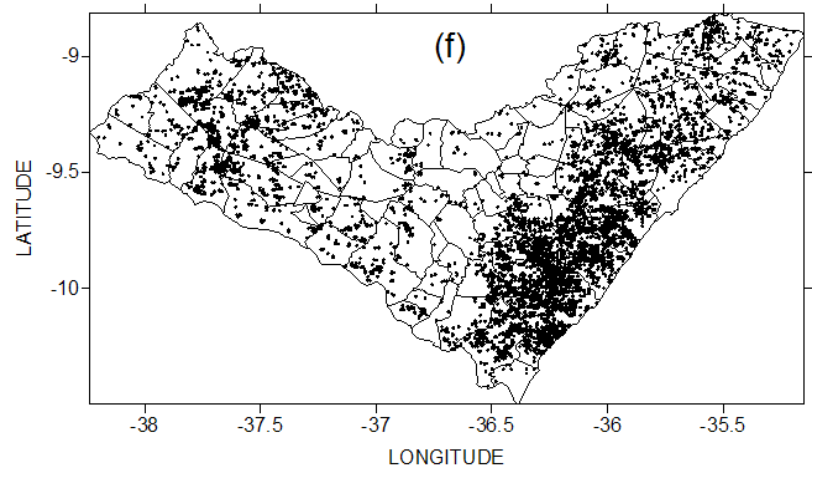

Figura 2 - Distribuição de acumulados bimestrais dos focos de queimadas no Estado de Alagoas, no período de 2004 a 2009. 

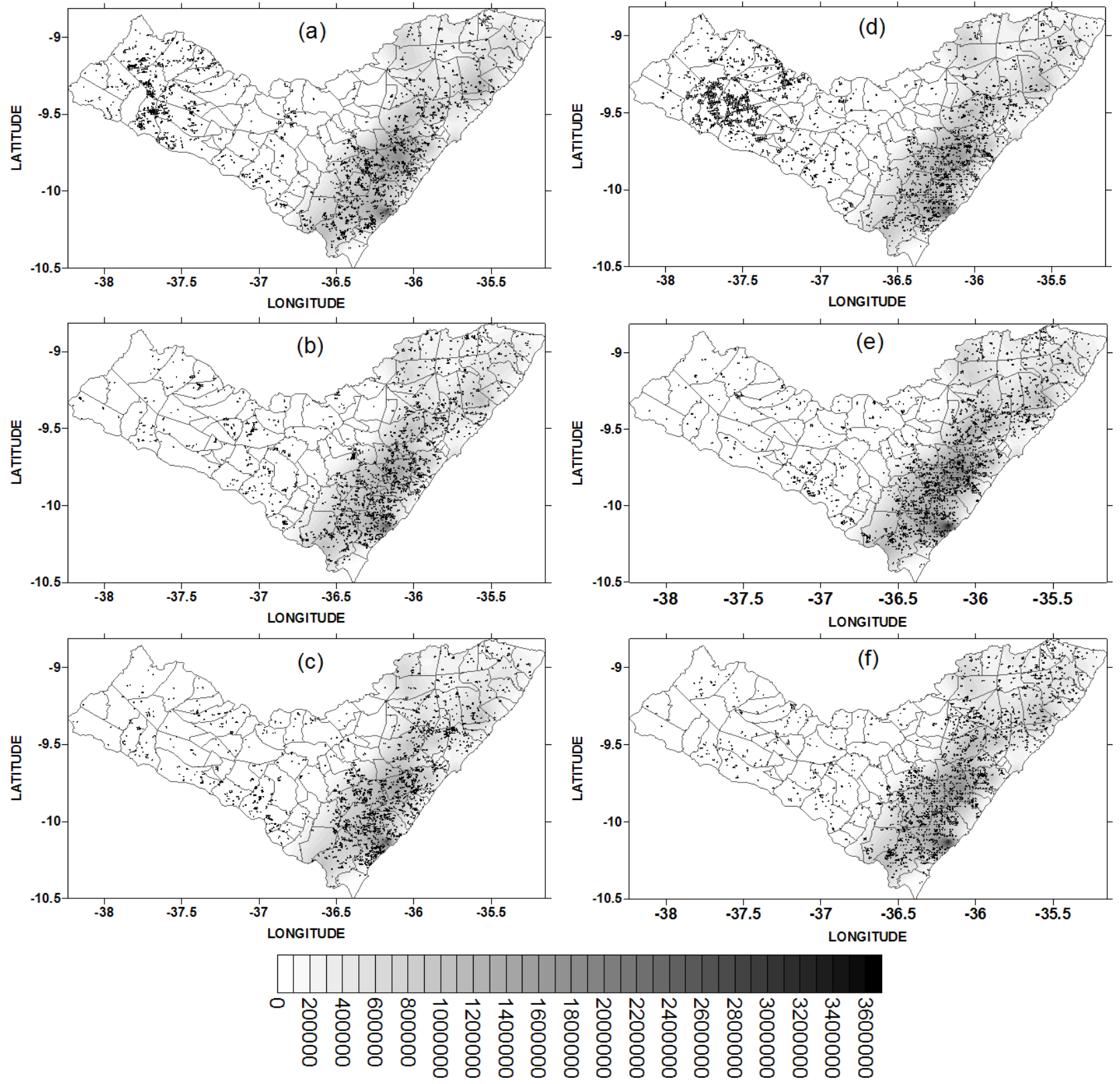

Figura 3 - Espacialização da produção anual de cana-de-açúcar, em toneladas, durante os anos de (a) 2004, (b) 2005, (c) 2006, (d) 2007, (e) 2008 e (f) 2009, em Alagoas, e os pontos de focos de queimadas nos respectivos anos.

ladas e os focos de queimadas nos respectivos anos. Em geral, dos 16.964 focos detectados entre 2004 a 2009 em Alagoas, o município de Coruripe teve o maior número de focos, com 2.034 casos $(13,3 \%)$, seguidos de Penedo, com 814 casos (5,4\%), São Miguel dos Campos, com 806 (5,3\%), Campo Alegre, com 677 $(4,5 \%)$ e Jequiá da Praia, com 639 (4,2\%). Percebe-se que os focos de queimadas detectados ocorreram principalmente na região do litoral alagoano, região canavieira. No caso de Coruripe, principal produtor de cana-de-açúcar, a cidade produz 2.690.040 toneladas (2005) e chega a 3.641.560 toneladas (2007) de cana-de açúcar, correspondendo a 14,5\% (321 focos) e
10,5\% (309 focos), respectivamente, do total de focos de queimadas em Alagoas.

Outros municípios como Jequiá da Praia, São Miguel dos Campos, Campo Alegre e Penedo, entre outros produtores de cana-de-açúcar, contribuem significativamente nos focos de queimadas nessa região. Em 2008, esses municípios contribuíram com $40 \%$ do total de focos em Alagoas, destacando-se Coruripe, com 15\% (429 focos), seguidos de São Miguel dos campos, com 7\% (196 focos) e Campo Alegre, com 6,3\% (176 focos). Observam-se, ainda, focos de queimadas em outros municípios, no entanto, na região do sertão alagoano, esses focos de queimadas devem-se à prá- 

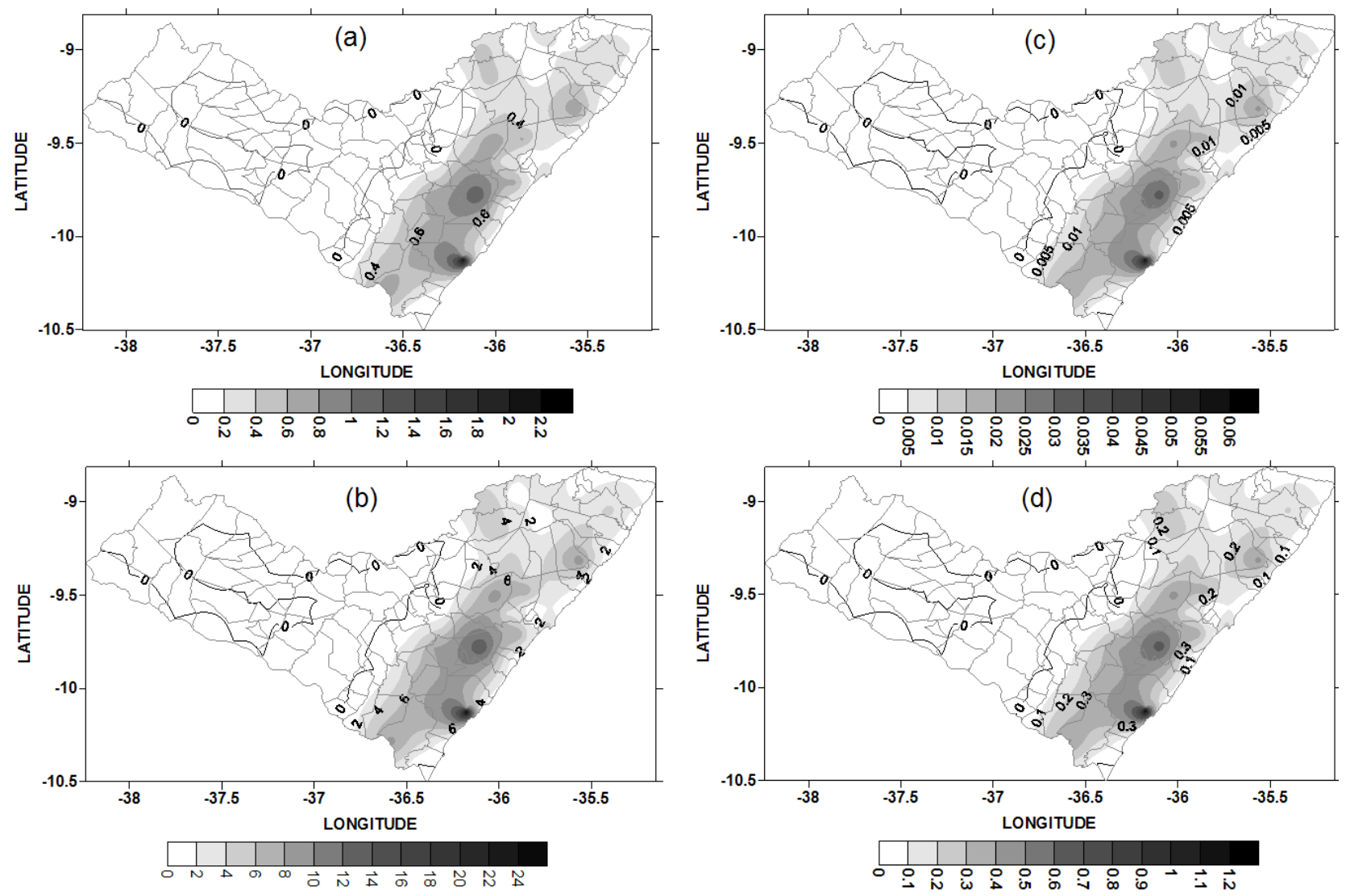

Figura 4 - Emissão média de (a) NO2, (b) monóxido de carbono, (c) N2O e (d) metano, em Gg/ano provenientes da queima de resíduos de cana-de-açúcar para Alagoas.

tica de queima da vegetação rasteira para produção agrícola sustentável e comercial, como feijão e milho.

Percebe-se que os maiores emissores de poluentes atmosféricos estão localizados na região sul do litoral. Além do mais, uma grande área de poluição é promovida por essa cultura, ocupando uma grande área que se estende do litoral até o agreste alagoano Figura 4 (a,b,c,d). Em média, Alagoas emitiu em torno de 17,6 Gg/ano, 270,0Gg/ano, 0,5Gg/ano, 9,9Gg/ano de $\mathrm{NO}_{2}, \mathrm{CO}, \mathrm{N}_{2} \mathrm{O}$ e $\mathrm{CH}_{4^{\prime}}$ respectivamente. Os municípios de Coruripe, São Miguel dos Campos, Atalaia e Penedo foram os maiores emissores desses poluentes para a atmosfera devido à cana-de-açúcar. O município de Coruripe apresentou as maiores emissões médio de $\mathrm{NO}_{2}(2,08 \mathrm{Gg} / \mathrm{ano}), \mathrm{CO}(24,5 \mathrm{Gg} / \mathrm{ano}), \mathrm{N}_{2} \mathrm{O}(0,06 \mathrm{Gg} /$ ano) e $\mathrm{CH}_{4}(1,16 \mathrm{Gg} / \mathrm{ano})$, seguido de São Miguel dos Campos, com 1,16 Gg/ano de $\mathrm{NO}_{2}$, 13,72 Gg/ano (CO), $\mathrm{N}_{2} \mathrm{O}(0,02 \mathrm{Gg} / \mathrm{ano})$ e $\mathrm{CH}_{4}(0,65 \mathrm{Gg} / \mathrm{ano})$ em Alagoas. Já Penedo emitiu cerca de três vezes menos que Coruripe.

É preocupante a contribuição de Alagoas na emissão de gases de efeito estufa para a atmosfera. Segundo Bowden (1990), o metano $\left(\mathrm{CH}_{4}\right)$ e o óxido nitroso $\left(\mathrm{N}_{2} \mathrm{O}\right)$ são mais eficientes que o $\mathrm{CO}_{2}$ na absorção da radiação de onda longa, em torno de 32 vezes e 150 vezes, respectivamente. Segundo o IPCC (1995), a cultura da cana-de-açúcar é responsável pela emissão anual de $11 \%$ de $\mathrm{CO}_{2}$ para a atmosfera. Outro ponto a ser observado, deve-se à população que habita essas regiões canavieiras ou regiões próximas a estas. Segundo Ribeiro (2008), os idosos, as crianças e os asmáticos têm sua saúde agravada pela queima da cana-de-açúcar. Para Lopes e Ribeiro (2006), a maior incidência de internações por doenças respiratórias estão em áreas canavieiras, a inalação do $\mathrm{NO}_{2}$ pela população dissolve-se nos tecidos e seu acúmulo nos brônquios tem provocado lesões nos pulmões e alterações no sistema imunológico (KURIYAMA, G. S.; MOREIRA, J. C. e SILVA, C. R. G., 1997; RUBSTEIN et al., 1991). Além disso, a deposição líquida e sólida do $\mathrm{NO}_{2}$ tem sido a causa da degradação e esterilização do solo, alteração do $\mathrm{pH}$, redução de produção na agricultura, acidificação de lagos e extermínio de árvores e florestas (ASHENDEN e BELL,1989; YAMULKI et al., 1997; DRISCOLL, et al., 1998; GRODZIŃSKAJURCZAKA e SZAREK-ŁUKASZEWSKAB, 1999). 


\section{Conclusões}

Investigando os focos de queimadas em Alagoas, detectados pelos satélites, foi constatado que as maiores incidências ocorreram na região canavieira entre os meses de setembro e fevereiro, período da colheita da cana-de-açúcar, sendo a prática da queima da palha um agente facilitador da colheita, e, no sertão, devido à climatologia da região. Constatou-se também que o município de Coruripe é o maior produtor de cana-de-açúcar e de focos.

Em relação à emissão de poluentes atmosféricos, os municípios de Coruripe e São Miguel dos Campos foram os maiores emissores de poluentes e de gases de efeito estufa em Alagoas.

Portanto, nas regiões produtoras de canade-açúcar em Alagoas, encontram-se as maiores incidências de focos de queimadas e, consequentemente, maiores emissões de poluentes atmosféricos e de gases de efeito estufa.

\section{Agradecimentos}

Agradecemos enormemente aos revisores deste artigo pelas contribuições e cordialidade, e a queridíssima amiga Ilka Cedrim pela revisão ortográfica e correções que elevam a representatividade deste artigo.

\section{Referências}

ASHENDEN, T. W.; BELL, S. A. Growth responses of three legume species exposed to simulated acid rain. Environmental Pollution, v.62,n¹, p. 21-29, 1989.

AZMI, S.Z., LATIF, M.T, ISMAIL, A.S., JUNENG, L.; JEMAIN, A.A., Trend and status of air quality at three different monitoring stations in the Klang. Valley, Malaysia. Air Qual. Atmos. \& Health, DOI: 10.1007/s11869-009-0051-1, 2009.

BOWDEN, R.D.;STEUDLER, P.A.; MELILIO, J.M; Annual nitrous oxide fluxes from temperate forest soils in the northeastern United States, Journal of Geophysical Research, v.95, p. 13997-14997, 1990.

CARDIM, A.H, Caracterização da estação de cultivo em Alagoas: análise temporal e espacial. Dissertação (Mestrado Meteorologia) - Universidade Federal de Alagoas - AL, p.51-65, 2003.
CARVALHO, A.L., Estação de cultivo baseada na precipitação pluvial diária e na ocorrência de períodos secos para a Região de Rio Largo, Alagoas. Dissertação (Mestrado Meteorologia) Universidade Federal de Alagoas - AL, 69p., 2010.

DUNCAN, B. N.; MARTIN, R. V.; STAUDT, A. C.; YEVICH, R.; LOGAN, J. A. Interannual and seasonal variability of biomass burning emissions constrained by satellite observations, J. Geophys. Res., 108(D2), 4100, doi:10.1029/2002JD002378, 2003.

FREITAS, S. R.; LONGO, K. M.; M. A. F. SILVA DIAS; SILVA DIAS P.L. Emissões de queimadas em ecossistemas da América do Sul. Estudos Avançados, v.19, n53, p. 167-185, 2005.

GRODZIŃSKA-JURCZAKA,M.;U,SZAREKŁUKASZEWSKAB, G. Evaluation of $\mathrm{SO}_{2}$ and $\mathrm{NO}_{2}$ -related degradation of coniferous forest stands in Poland. The Science of the Total Environment, 241, p. 1-15, 1999.

\section{IBGE, Instituto Brasileiro de Geografia e}

Estatística. Produção Agrícola Municipal, disponível em: <http://www.sidra.ibge.gov.br/bda/ tabela/listabl.asp?c=1612\&z=t\&o=11>. Acessado em 03 Maio de 2013.

KURIYAMA, G. S.,MOREIRA, J. C.SILVA, C. R. S. Exposição ocupacional ao dióxido de nitrogênio $\left(\mathrm{NO}_{2}\right)$ em policiais de trânsito na cidade do Rio de Janeiro. Cad. Saúde Públ., Rio de Janeiro, v.13, n²4, p. 677-683, out-dez, 1997.

LARA, L.L.; ARTAXO, P.; MARTINELLI, L.A.; CAMARGO, P.B.; VICTORIA, R.L.; FERRAZ, E.S.B.. Properties of aerosols from sugar-cane burning emissions in Southeastern Brazil, Atmos. Environ., Vol.39, 26, p. 4627-4637, 2005

LIMA, M. A.; LIGO, M. A. V.; PESSOA, M. C. P. Y.; NEVES, M. C.; CARVALHO, E. C. de Emissões de gases de efeito estufa na queima de resíduos agrícolas. Brasília: Ministério da Ciência e Tecnologia: Embrapa, 2010. Segundo Inventário Brasileiro de Emissões e Remoções Antrópicas de Gases de Efeito Estufa - Relatórios de referência.

REMY, S; NAWROT, T; FIERENS, F.; PETIT, P.; VANDESTRAETEN, P.; NEMERY, B.; BOULAND, C., Health impact of urban air pollution in Belgium, Air Qual. Atmos. \& Health, DOI: 10.1007/s11869-010-0078-3, 2010. 
RIBEIRO, H. Queimadas de cana-de-açúcar no Brasil: efeitos à saúde respiratória. Rev. Saúde Pública, v.42, n², p. 370-376, 2008.

RONQUIM, C. C. Queimadas na colheita da cana-de-açúcar: impactos ambientais, sociais e econômicos. Campinas, SP: Embrapa Monitoramento por Satélite, 2010. 45 p. (Documentos, 77).

RUBSTEIN, I.; REISS, T. F.; BIGBY, B. G.; STITES, D. P.;BOUSHEY, H. A.,. Effects of 0,60 ppm nitrogen dioxide on circulating and broncho alveolar lavage lymphocyte phenotypes in health subjects. Environmental Research, 55:18-30, 1991.

SANTOS, S.S. (2008) O cultivo da Cana-deaçúcar no estado de Alagoas: uma análise comparativa da mecanização no estado de São Paulo. Dissertação de Mestrado. Centro de Desenvolvimento Sustentável. 100p, Universidade de Brasília, 2008.

YAMULKI, S.; HARRISON, R.M.; GOULDING, K. W. T.; WEBSTER, C. P. $\mathrm{N}_{2} \mathrm{O}, \mathrm{NO}$ and $\mathrm{NO}_{2}$ fluxes from a grassland: Effect of soil ph. Soil Eiol. Biochem. V.29, nº, p. 1199-1208. 1997. 